\title{
Wavelet transform applied to pulse thermographic data for detection of subsurface defects in aluminum structures
}

\author{
Yoonjae Chung*, Seungju Lee* and Wontae Kim** \\ *Department of Mechanical \& Automotive Engineering, Kongju National University, 1223- \\ 24 Cheonan-daero, Seobuk-gu, Cheonan-si, Chungcheongnam-do, 31080, South Korea
}

\begin{abstract}
:
In this study, we focused on pulse infrared thermography as a non-destructive testing method for detection of subsurface defects in aluminum structures. In the present investigation, a square shaped $(180 \mathrm{~m} * 180 \mathrm{~mm})$ aluminum specimen with $10 \mathrm{~mm}$ thickness and artificial defects with circular cutouts of varying depth and diameter at the back side was manufactured. The sample was excited at several modulation frequencies by a sinusoidal heat flux, and a thermal infrared camera was utilized for monitoring of surface temperature of a thermal wave that propagated into the sample. Wavelet transform was applied to compute phase angle data from the temperature-time history of each pixel for the assessment of the defects. A phase image was calculated using a Fourier transform and the Wavelet transform. Image processing software MATLAB and Thermofit Pro were used to compute the phase image. The investigation into the effects of wavelet parameters; scale and shift, modulation frequencies, inclusion sizes, and depths on the phase contrast was conducted and discussed.
\end{abstract}

\section{Acknowledgement}

This work was supported by National Research Foundation of Korea (NRF) grant funded by the Ministry of Education, Science and Technology (NRF-2019R1F1A1061328). 